\title{
CONSTRUCTION OF CONVEX SETS IN NEGATIVELY CURVED MANIFOLDS
}

\author{
ALBERT BORBÉLY
}

(Communicated by Jonathan M. Rosenberg)

\begin{abstract}
It was proved by Choi that one can solve the Dirichlet problem at infinity for simply connected negatively curved manifolds by constructing appropriate convex sets. All the known constructions, it seems, inherently need some kind of growth condition on the curvature; therefore, it is interesting to find new ways to construct convex sets in negatively curved manifolds. In this paper we give a new way to construct convex sets from sets we call $\varepsilon$-almostconvex. From the point of view of this problem this can be considered as a natural generalization of convexity.
\end{abstract}

\section{INTRODUCTION}

Anderson and Sullivan (cf. [A, S]) proved that the Dirichlet problem at infinity for simply connected negatively curved manifolds is solvable provided that the curvature satisfies $-a^{2}<K<-b^{2}$, for some constants $a, b$. This result was sharpened in [B] by replacing the lower curvature bound with an exponential growth condition using the method of Anderson-Choi. It suggests that the lower bound might be dispensed with altogether. This method hinges upon Choi's theorem (cf. [C]), which states:

Theorem (Choi). Let $N^{n}$ be a simply connected negatively curved manifold with curvature bounded away from 0 . Denote by $S^{n-1}(\infty)$ the ideal boundary, and suppose that for every $p, q \in S^{n-1}(\infty)$ there are disjoint open convex sets $V, W$ which separate $p, q$ in the cone topology of $N^{n} \cup S^{n-1}(\infty)$ (cf. [EbO, A]).

Then the Dirichlet problem at infinity for $\Delta$ is uniquely solvable for any continuous function on the ideal boundary.

The method of constructing convex sets in [A, B], it seems, inherently needs some kind of growth condition on the curvature; therefore, it is interesting to find new ways to construct convex sets in negatively curved manifolds.

Let $N^{n}$ be a simply connected negatively curved manifold. First we define the notion of an almost-convex set.

Definition. We call an open set $A \subset N^{n}$ almost-convex if every point $p \in \partial A$ can be touched by a horosphere from the outside; that is, there exists an open horoball $B$ such that $A \cap B=\varnothing$ and $p \in \partial A \cap \partial B$.

Received by the editors April 29, 1991.

1991 Mathematics Subject Classification. Primary 53C20. 
We call an open set $A \subset N^{n} \varepsilon$-almost-convex if $\partial A$ is a $C^{3}$ hypersurface with the following property: for every $p \in \partial A$ denote by $U$ the 2 nd fundamental form of $\partial A$ with respect to the outer normal and by $V$ the 2 nd fundamental form of the touching horosphere from the outside with respect to the inner normal. We require that

$$
U(X, X) \geq V(X, X)+\varepsilon\|X\|^{2}
$$

for every $X \in T_{p} \partial A$.

In the sequel for two symmetric matrices $A, B$ we write $A>B$ or $A \geq B$ if $A-B$ is positive definite or positive semidefinite, respectively. We use the sign convention for the 2 nd fundamental form such that the 2 nd fundamental form of a sphere with respect to the outer normal is positive definite. With this definition we now have

Theorem 1.1. Let $N^{n}$ be a simply connected negatively curved manifold and $A \subset N^{n}$ be an open $\varepsilon$-almost-convex set. Let $A_{t}=\left\{p \in N^{n}: \operatorname{dist}(p, A) \leq t\right\}$. Then for $t>n / \varepsilon, A_{t}$ is convex.

Remark. With this theorem one can generalize Choi's theorem by simply replacing the convex sets $V_{x}, V_{y}$ in the statement of his theorem by $\varepsilon$-almost-convex sets.

The proof is based on the stability properties of the Riccati equation which describes the evolution of parallel hypersurfaces. As an easy consequence of the method we can also prove the following lemma.

Lemma 1.2. Let $N^{n}$ be a simply connected complete negatively curved manifold and $H$ be a horosphere. Let $p \in H$ and $S_{r}$ a sphere of radius $r$ inside $H$ which touches $H$ at $p$. Then

$$
V-\frac{n}{r} \mathrm{Id} \leq U \leq V
$$

where $V$ and $U$ are the 2nd fundamental forms of the horosphere $H$ and the sphere $S_{r}$ with respect to the inner normal, respectively.

\section{Proofs}

Set $S=\partial A$, and denote by $S_{t}$ the parallel hypersurface (outside $A$ ) to $S$ at distance $t \geq 0$. Let $p \in S$ and $\gamma$ be the geodesic emanating from $p$ in the direction of the outer normal. Denote by $U(t)$ the 2 nd fundamental form of $S_{t}$ with respect to $\gamma^{\prime}(t)$ and by $V(t)$ the 2nd fundamental form of the horosphere centered at $\gamma(\infty)$ (touching $A_{t}$ from the outside) with respect to $\gamma^{\prime}(t)$. We know (cf. [HH, Eb]) that the horosphere is $C^{2}$ so it has a 2 nd fundamental form.

We have to prove that for $t>n / \varepsilon, U(t)$ is positive semidefinite.

With the stated sign convention, we have the evolution equation for the 2 nd fundamental forms of parallel hypersurfaces.

$$
U^{\prime}+U^{2}+R=0
$$

where $R(X)=R\left(\gamma^{\prime}, X\right) \gamma^{\prime}$ is the curvature tensor and $U^{\prime}$ means the covariant derivative of $U=U(t)$ along $\gamma$. The same equation is true for $V=V(t)$, which is negative definite with this choice of the 2 nd fundamental form. 
We note that if $R$ is a symmetric matrix-valued function then every solution $U$ of (2.1) with symmetric initial value is symmetric.

We know that $(2.1)$ has two special all time (i.e., $(-\infty, \infty)$ ) solutions. One is $V$, and the other is $W$, the 2 nd fundamental form of the horosphere centered at $\gamma(-\infty)$ with respect to $\gamma^{\prime}$. This is positive definite. We will show that $W$ is the stable and $V$ is the unstable solution of $(2.1)$.

Our main tool is the comparison theorem for the Riccati equation (cf. [EH]).

Comparison Theorem. Consider the Riccati equations

$$
B_{j}^{\prime}+B_{j}^{2}+R_{j}=0, \quad j=1,2,
$$

where $B_{j}, R_{j}$ are smooth functions of $n \times n$ symmetric matrices. Let $B_{j}$ be solutions of $\left(R_{j}\right)$ on $\left[0, t_{j}\right)$ with maximal $t_{j}$. Suppose that $R_{1} \geq R_{2}$ and $B_{2}(0) \geq B_{1}(0)$.

Then $t_{2} \geq t_{1}$ and $B_{2}(t) \geq B_{1}(t)$ for all $t \in\left[0, t_{1}\right)$.

Because $V$ is an all time solution of $(2.1)$ this immediately gives that $U(t)$ in the theorem exists for all $t>0$; that is, the parallel surface $S_{t}$ never degenerates, i.e., it is always $C^{2}$.

We start our investigation of (2.1) with some elementary facts. Suppose now that $R$ is some negative semidefinite matrix-valued function on $(-\infty, \infty)$, which does not necessarily come from a curvature tensor of a Riemannian manifold.

Proposition 2.3. Let $U_{i} \geq 0, i=1,2$, be positive semidefinite solutions of the Riccati equation (2.1) on the interval $[s, \infty)$. Then the distance between $U_{1}$ and $U_{2}$ is decreasing monotonically to zero; that is,

$$
\frac{d}{d t}\left\|U_{1}-U_{2}\right\|^{2} \leq 0 \text { and }\left\|U_{1}-U_{2}\right\|^{2} \rightarrow 0,
$$

where, for a matrix $A$, we define the norm by $\|A\|^{2}=\operatorname{tr}\left(A A^{T}\right)$.

Proof. Define the function

$$
f(t)=\left\|U_{1}-U_{2}\right\|^{2}=\operatorname{tr}\left(\left(U_{1}(t)-U_{2}(t)\right)\left(U_{1}(t)-U_{2}(t)\right)^{T}\right) .
$$

Differentiating it gives us

$$
f^{\prime}(t)=2 \operatorname{tr}\left(\left(U_{1}-U_{2}\right)^{\prime}\left(U_{1}-U_{2}\right)\right) .
$$

From differential equation (2.1) we have

$$
f^{\prime}(t)=-2 \operatorname{tr}\left(\left(U_{1}^{2}-U_{2}^{2}\right)\left(U_{1}-U_{2}\right)\right) .
$$

Applying elementary properties of the trace we have

$$
f^{\prime}(t)=-2 \operatorname{tr}\left(\left(U_{1}+U_{2}\right)\left(U_{1}-U_{2}\right)^{2}\right) .
$$

Since $U_{1}, U_{2} \geq 0$, we get $f^{\prime}(t) \leq 0$. To estimate $f^{\prime}(t)$ we pick an orthonormal system of eigenvectors $e_{1}, e_{2}, \ldots$ for $U_{1}-U_{2}$ with corresponding eigenvalues $\lambda_{1}, \lambda_{2}, \ldots$. Then

$$
f^{\prime}(t)=-2 \sum \lambda_{i}^{2}\left(\left\langle U_{2} e_{i}, e_{i}\right\rangle+\left\langle U_{1} e_{i}, e_{i}\right\rangle\right)
$$

but $\left\langle U_{1} e_{i}, e_{i}\right\rangle-\left\langle U_{2} e_{i}, e_{i}\right\rangle=\lambda_{i}$, so it gives us, via the Hölder inequality,

$$
f^{\prime}(t) \leq-2 \sum\left|\lambda_{i}\right|^{3} \leq-2\left(n^{-1 / 3} f\right)^{3 / 2}=-2 n^{-1 / 2} f^{3 / 2},
$$


which implies that $f(t) \rightarrow 0$ as $t \rightarrow \infty$. This concludes the proof of Proposition 2.3.

Proposition 2.4. Equation (2.1) has a positive semidefinite solution $W$ and a negative semidefinite solution $V$ for all time (i.e., on $(-\infty, \infty)$ ).

Proof. First we construct $W$, the positive definite all time solution. Let $U_{t}$ be a solution of $(2.1)$ on $[t, T)$ with maximal $T$ and with initial condition $U_{t}(t)=0$. Comparing this with the zero solution for $R \equiv 0$ implies that $U_{t}(s)$ is defined for all $s>t$ and $U_{t} \geq 0$ on $[t, \infty)$.

We now define $W$ by

$$
W(s)=\lim _{t \rightarrow-\infty} U_{t}(s) .
$$

The comparison theorem tells us that $U_{t}(s)$ is monotone increasing as $t \rightarrow-\infty$ so that the limit exists. To show that $W(s)$ is finite we need an argument which we will use later in Proposition 2.5; therefore, we only sketch it here.

Fix $s \in \mathbf{R}$, and let

$$
\Lambda=\max _{x \in[s-2, s]}\{|\lambda(x)|, 1\}
$$

where $\lambda(x)$ is the smallest eigenvalue of $R(x)$. We can easily estimate the solution $\widetilde{U}$ of $(2.1)$ on the interval $[s-2, s]$ where $\widetilde{R} \equiv-\Lambda$ Id and the initial condition is $\widetilde{U}(s-2)=\infty$ Id (i.e., arbitrarily large initial condition). A similar argument we use at the end of the proof of Proposition 2.6 shows that $\widetilde{U}(s)<$ $2 \Lambda \mathrm{Id}$. Using the comparison principle, we have $U_{t}(s)<\widetilde{U}(s)<2 \Lambda$ Id for any $t<s-2$.

The next step is to prove uniform convergence on compact sets. For a compact set $K \subset(-\infty, \infty)$ choose $s_{0}<K$, that is, $s_{0}<k$ for any $k \in$ $K$. We know that $U_{t}\left(s_{0}\right)$ is monotonically increasing and bounded as $t \rightarrow$ $\infty$. Therefore for any $\varepsilon>0$ there is a $t_{0}$ such that for every $t_{1}, t_{2}<t_{0}$, $\left|U_{t_{1}}\left(s_{0}\right)-U_{t_{2}}\left(s_{0}\right)\right|<\varepsilon$, which means via Proposition 2.3 that for any $k \in K$, $\left|U_{t_{1}}(k)-U_{t_{2}}(k)\right|<\varepsilon$, that is, $U_{t} \rightarrow W$ uniformly on $K$ as $t \rightarrow-\infty$; so, using (2.1) we have $U_{t}^{\prime} \rightarrow G$ uniformly on $K$, which in turn implies that $W$ is differentiable, $W^{\prime}=G$, and $W$ is a positive semidefinite solution of (2.1).

To construct $V$, we solve (2.1) backwards. Consider the matrix-valued equations on the real line

$$
Y^{\prime}+Y^{2}+\widetilde{R}=0,
$$

where $\widetilde{R}(t)=R(-t) . \widetilde{R} \leq 0$, so we have a positive definite solution $\widetilde{W} \geq 0$ on $(-\infty, \infty)$. Set $V(t)=-\widetilde{W}(-t)$. Then it is easy to check that this is a negative semidefinite solution of $(2.1)$, which proves the proposition.

Remark. The uniqueness of $W$ and $V$ will follow from Propositions 2.5 and 2.6. $V$.

In Propositions 2.5 and 2.6 we examine the stability properties of $W$ and

Proposition 2.5. Suppose that $V(t)$ is a negative semidefinite solution of (2.1) on $(-\infty, \infty), U(0) \leq V(0)$, and $\operatorname{tr} U(0)+\varepsilon<\operatorname{tr} V(0)$ for some $\varepsilon>0$. Let $U(t)$ be the matrix-valued solution of $(2.1)$ with initial value $U(0)$ on $[0, T)$ where $T$ is maximal. Then $T \leq n / \varepsilon$. 
Proof. The comparison theorem tells us that $U \leq V \leq 0$ for all $t \in[0, T)$; that is, we can write $U=V-A$ where $A \geq 0$. Substituting $U$ and $V$ into (2.1) and subtracting one from the other gives

$$
(V-U)^{\prime}=U^{2}-V^{2} \text {. }
$$

Taking the trace we have

$$
[\operatorname{tr}(V-U)]^{\prime}=\operatorname{tr}\left(U^{2}-V^{2}\right)=\operatorname{tr}\left(U^{2}+V^{2}-2 V U\right)+2 \operatorname{tr}\left(V U-V^{2}\right) .
$$

Now $\operatorname{tr} V U=\operatorname{tr} V^{2}-\operatorname{tr} V A$. So $A \geq 0$ and $V \leq 0$ implies that $\operatorname{tr} V A \leq$ 0 (choose $e_{i}$ to be the eigenvectors of $A$; then $\operatorname{tr} V A=\sum\left\langle V A e_{i}, e_{i}\right\rangle=$ $\sum \lambda_{i}\left\langle V e_{i}, e_{i}\right\rangle \leq 0$, where $\lambda_{i} \geq 0$ are the corresponding eigenvalues); that is, $\operatorname{tr} V U \geq V^{2}$. Substituting this into (2.4) we have, via the Hölder inequality,

$$
[\operatorname{tr}(V-U)]^{\prime} \geq \operatorname{tr}(V-U)^{2} \geq \frac{1}{n}[\operatorname{tr}(V-U)]^{2} .
$$

Set $f(t)=\operatorname{tr}(V(t)-U(t))$. Then (2.5) becomes

$$
f^{\prime} \geq \frac{1}{n} f^{2}, \quad f(0) \geq \varepsilon
$$

It is easy to show that (2.6) does not have a solution on $[0, \eta+n / \varepsilon)$ for any $\eta>0$. Let $g(t)$ be the solution of the differential equation $g^{\prime}=\frac{1}{n} g^{2}$ with $g(0)=f(0)$. Then $f(t) \geq g(t)$ whenever $f(t)$ exists. The solution of this differential equation gives us that

$$
g(t)=\frac{f(0) n}{n-t f(0)},
$$

so $g \rightarrow \infty$ as $t \rightarrow n / f(0) \leq n / \varepsilon$. This proves Proposition 2.5.

Proposition 2.6. Suppose that $V$ is a negative semidefinite solution of (2.1) and $W$ is a positive semidefinite solution of $(2.1)$ for all time (i.e., on $(-\infty, \infty)$ ). If $U(0) \geq V(0)+\varepsilon$ Id then $(2.1)$ has a solution on $[0, \infty)$ with initial value $U(0)$, $U(t) \geq 0$ for $t>n / \varepsilon$ and $\|U-W\| \rightarrow 0$ as $t \rightarrow \infty$.

Proof. The comparison theorem tells us that $U$ is defined on $[0, \infty)$. Set $T=n / \varepsilon$, and let $\varphi$ be a cut-off function such that $0 \leq \varphi \leq 1, \varphi(s)=1$ for $s<T$, and $\varphi(s)=0$ for $s>T+a$ where $a$ is a small positive number.

Consider the equation

$$
Y^{\prime}+Y^{2}+R_{1}=0, \quad \text { where } R_{1}=\varphi R .
$$

Proposition 1 shows that there is a negative semidefinite solution $Y$ of $(2.7)$ for all time (i.e., on $(-\infty, \infty))$. From the construction of $Y$ or from Proposition 2.5 , it is obvious that $Y=0$ on $[T+a, \infty)$. In case for some $s \in[T+a, \infty)$, $\operatorname{tr} Y(s)<0$, Proposition 2.5 would imply that $Y \rightarrow-\infty$ within $n / \operatorname{tr} Y(s)$ time.

Using the comparison principle backwards, that is, to the equations

$$
X^{\prime}+X^{2}+\widetilde{R}_{i}=0, \quad \text { where } \widetilde{R}_{0}(t)=-R(t), \widetilde{R}_{0}(t)=-R_{1}(t),
$$

we know if $X_{1}(-T-1) \leq X_{0}(-T-1)$ and $\widetilde{R}_{1} \geq \widetilde{R}_{0}$ then $X_{1}(0) \leq X_{0}(0)$. Setting $X_{1}(-t)=-Y(t)$ and $X_{0}(t)=-V(-t)$ we now have that $X_{1}(-T-1) \leq$ $X_{0}(-T-1)$, and therefore $Y(0) \geq V(0)$; but because $R_{1}=R$ on $[0, T]$, Proposition 2.5 implies that $\operatorname{tr} Y(0)-\operatorname{tr} V(0) \leq \varepsilon$, otherwise $V \rightarrow-\infty$ within $n / \varepsilon$ time, which is a contradiction. 
We now have the inequality

$$
V(0) \leq Y(0) \leq V(0)+\varepsilon \text { Id } \leq U(0) .
$$

Applying the comparison principle to $Y$ and $U\left(Y(0) \leq U(0)\right.$ and $R_{1} \geq$ $R$ ) yields that, for every $t>0, U(t) \geq Y(t)$, that is, $U$ becomes positive semidefinite for $t>T+a$. Since $a$ is arbitrarily small, $U$ becomes positive semidefinite for $t>T$.

Now Proposition 2.3 shows that $\|U-W\|^{2} \rightarrow 0$ as $t \rightarrow \infty$.

This concludes the proof of Proposition 2.6.

Proof of Theorem 1.1. Applying Proposition 2.6 to evolution equation (2.1) of parallel hypersurfaces proves the theorem immediately.

Proof of Lemma 1.2. The inequality $U \leq V$ is trivial. To prove the other inequality denote by $\gamma$ the geodesic emanating from $p$ in the direction of the inner normal (it is centered at $\gamma(\infty)$ ). Let $V(t)$ and $U(t)$ be the 2 nd fundamental forms of the parallel horosphere and sphere, respectively, at $\gamma(t)$ with respect to $\gamma^{\prime}(t)$. We know that $U(t) \leq V(t) \leq 0, U(t)$ is defined on $[0, r)$, and they both satisfy the Ricatti equation

$$
X^{\prime}+X^{2}+R=0
$$

where $R \leq 0$ is the curvature tensor of $N^{n}$. We know that $V(t)$ is defined on $[0, \infty)$, so if $U(0) \nsupseteq V(0)-\left(a+\frac{n}{r}\right)$ Id then $\operatorname{tr} U+a+\frac{n}{r}<\operatorname{tr} V$; that is, according to Proposition $2.5 U$ is defined at most on the interval $[0, n /(a+n / r))$, which is a contradiction. Now letting $a$ go to zero proves the lemma.

\section{REFERENCES}

[A] M. T. Anderson, The Dirichlet problem at infinity for manifolds of negative curvature, J. Differential Geom. 18 (1983), 701-721.

[BA] W. Ballmann, On the Dirichlet problem at infinity for manifolds of nonpositive curvature, Forum Math. 1 (1989), 201-213.

[B] A. Borbély, A note on the Dirichlet problem at infinity for negatively curved manifolds, Proc. Amer. Math. Soc. 114 (1992), 865-872.

[C] H. I. Choi, Thesis, Univ. of California, Berkeley, 1982.

[Eb] P. Eberlein, Busemann functions are $C^{2}$ (unpublished).

[EbO] P. Eberlein and B. O’Neill, Visibility manifolds, Pacific J. Math. 46 (1973), 45-109.

[EH] J.-H. Eschenburg and E. Heintze, Comparison theory for Riccati equations, Manuscripta Math. 68 (1990), 209-214.

[HH] E. Heintze and H. I. Hopf, Geometry of horospheres, J. Differential Geom. 12 (1977), 481491.

[S] D. Sullivan, The Dirichlet problem at infinity for a negatively curved manifold, J. Differential Geom. 18 (1983), 723-732.

Department of Mathematics, University of Notre Dame, Notre Dame, Indiana 46556 Current address: Hataror ut 19/c, 1122 Budapest, Hungary 